\title{
Impact of modified ultrafiltration on Cardiac Surgery-Associated Acute Kidney Injury
}

Hani Mohammed Ahmed Raslan*1, Ahmed Al-saied Abdul Rahman², Huda Fahmy Mahmoud ${ }^{1}$

${ }^{1}$ Department of Anaesthesia and Intensive Care - Faculty of Medicine - Sohag University, ${ }^{2}$ Department of

Anaesthesia and Intensive care- Faculty of Medicine - Aswan University

*Corresponding author: Hani Mohammed Ahmed Raslan, Mobile: (+20) 01014734266, E-Mail: hani.raslan@ymail

\begin{abstract}
Background: Cardiac surgery-associated acute kidney injury (CSA-AKI) is a frequent happening affecting nearly $40 \%$ of the cases undergoing cardiac surgery. This is associated with increased hospital length of stay and mortality. Objective: The aim of our study was to investigate the relationship impact of modified ultrafiltration on cardiac surgery-associated acute kidney injury (CSA-AKI).

Patients and Methods: This prospective observational study was conducted over a 6-month period (February to July 2017) at Cardiothoracic Surgery Unit, Kasr AL-Ainy Hospitals. The study included 40 patients undergoing routine valve replacement surgery using cardiopulmonary bypass.

Results: There was no significant difference $(\mathrm{P}>0.05)$ between AKI and non-AKI patients in pre-operative levels while the postoperative serum creatinine levels were significantly higher $(\mathrm{P}<0.04)$ in AKI than non-AKI patients in the modified ultrafiltration (MUF) group. The pre-operative and $2 \mathrm{~h}$ post-operative S. NGAL was significantly higher in AKI than non-AKI patients in the MUF group were. Duration of CPB was significantly $(\mathrm{P}<0.001)$ prolonged in the AKI than the non-AKI patients. While, there was no significant difference regarding aortic crossclamp time. There was a significant longer ICU stay and duration of mechanical ventilation in AKI than non-AKI patients.
\end{abstract}

Conclusion: Modified ultrafiltration after cardiac surgery is associated with a lower prevalence of morbidity and mortality. Serum Neutrophil Gelatinase-Associated Lipocalin (NGAL) can be used as an early plasma biomarker predictor for AKI in patients undergoing heart surgery.

Keywords: Modified ultrafiltration, Acute kidney injury biomarkers, Valvular heart surgery.

\section{INTRODUCTION}

Cardiac surgery-associated acute kidney injury (CSA-AKI) is a common and serious postoperative complication of cardiac surgery that employs cardiopulmonary bypass (CPB), and it is the second most common cause of AKI in the intensive care unit (ICU) ${ }^{(\mathbf{1})}$. CSA-AKI is characterized by an abrupt deterioration in kidney function following cardiac surgery as evidenced by a reduction in the glomerular filtration rate. Importantly, this deterioration may not be detected in the first $24-48 \mathrm{~h}$ using conventional monitoring by serum creatinine (s.cr) levels because of the dilutional effects of the CPB pump prime ${ }^{(2)}$.

CSA-AKI is caused by a variety of factors, including exogenous and endogenous toxins, metabolic abnormalities, ischemia and reperfusion injury, neurohormones activation, inflammation and oxidative stress ${ }^{(2)}$. Postoperative kidney function deterioration has been shown to be an important predictor of morbidity and mortality ${ }^{(3)}$.

Modified ultrafiltration has been explored as a potential intervention to mitigate AKI risk in cardiac surgery ${ }^{(4)}$. Conversely, modified ultrafiltration may have an adverse consequence on kidney injury, mechanistically related to fluid shift imbalances and renal hypoperfusion. A few relatively small studies have explored the short-term risks associated with modified ultrafiltration, and have assessed kidney injury in a number of ways, including renal insufficiency, creatinine clearance $(\mathrm{CrCl})$, and elevated postoperative creatinine ${ }^{(5)}$.

Modified ultrafiltration has been used to treat the hemodilutional effects of cardiopulmonary bypass circuits. The impact of modified ultrafiltration on risk of acute kidney injury (AKI) remains uncertain. Failure to perform modified ultrafiltration with resultant hemodilution or fluid overload can potentially result in kidney injury through tissue edema, as the kidneys are encapsulated organs (6). Modified ultrafiltration has recently increasingly come into favor. The technique entails removal of water and low-molecular-weight substances under a hydrostatic pressure gradient after separation from cardiopulmonary bypass.

This method has been demonstrated to induce hemoconcentration and reduce bleeding and total body water accumulation ${ }^{(7)}$.

Because of the properties of counteracting tissue edema and eliminating inflammatory mediators, further observations have attributed to modified ultrafiltration the ability to improve acute kidney injury (AKI) function and to attenuate morbidity after cardiac surgery ${ }^{(7,8)}$.

\section{AIM OF THE WORK}

This article is an open access article distributed under the terms and conditions of the Creative Commons Attribution (CC BY-SA) license (http://creativecommons.org/licenses/by/4.0/) 
The aim of our study was to investigate the relationship impact of modified ultrafiltration on cardiac surgery-associated acute kidney injury (CSAAKI).

\section{PATIENTS AND METHODS}

This prospective observational study was conducted over a 6-month period (February to July 2017) at Cardiothoracic Surgery Unit, Kasr AL-Ainy Hospitals. The study included 40 patients undergoing routine valve replacement surgery using cardiopulmonary bypass.

Inclusion criteria: Adult patients $>16$ years. Patients undergoing open valvular heart surgery using CPB.

Exclusion criteria: Patients < 16 years. Emergency or redo surgery. ASA Class IV patients. Preoperative renal dysfunction or chronic renal disease. Poor preoperative ventricular function.

The patients were randomly allocated into two groups:

- Group1: MUF group

- Group 2: Non-MUF group

Ethical approval and written informed consent:

An approval of the study was obtained from Aswan University Academic Ethical Committee. Every patient signed an informed written consent for acceptance of the operation.

\section{Anesthesia Technique:}

- Patients scheduled for cardiac surgery are usually fully investigated for their cardiac status. However, the presence of non-cardiac medical illnesses and routine general anesthetic assessment should not be overlooked including routine laboratory investigation and arrangements of blood products.

- All patients were premeditated with 5-10 mg oral midazolam the night before surgery.

- After transferring the patient to preoperative holding area, arterial line (radial artery) was introduced in the patient non-dominant hand after reviewing duplex study.

- Induction was made using propofol $(1-2 \mathrm{mg} / \mathrm{kg})$, atracurium (04mg kg), fentanyl (50-microgram increments).

- After tracheal intubation the patients were mechanically ventilated to give a tidal volume (6$8 \mathrm{ml} \mathrm{kg}$ )

- Maintenance of anesthesia achieved by isoflurane in $60 \%$ oxygen and atracurium infusion $(0.3$ to $0.6 \mathrm{mg} / \mathrm{kg} /$ hour).

- $\mathrm{CPB}$ was conducted on all cases using membrane oxygenator, non-occlusive roller pumps and priming of all cases was done with crystalloids. Myocardial protection was achieved using a cardioplegia solution in a dose of $20 \mathrm{ml} / \mathrm{kg}$ and modified ultrafiltration was performed after getting off bypass for about15-20 minutes. Patients were transferred to the ICU intubated.

\section{Technique of modified ultrafiltration:}

In this method, the arterial line is connected to the inlet of the ultrafilter and the venous line is connected to the outlet of the ultrafilter in the CPB circuit. The inlet of the filter was clamped throughout the CPB. As the patient is separated from the CPB, the clamp is removed from the inlet of the filter, allowing the blood to flow through the arterial line to the filter $(10-15 \mathrm{~mL} / \mathrm{kg} / \mathrm{min})$, and finally from the cardioplegic cannula, as a venous line returns to the right atrium.

\section{Indicator detection:}

NGAL and S.cr levels were detected using ELISA according to the manufacturer's instructions. In brief: i) blood stored at $-80^{\circ} \mathrm{C}$ was thawed at room temperature $\left(20^{\circ} \mathrm{C}\right)$, ii) samples and standards were diluted (dilution ratio 1:5) and transferred to the plate, iii) samples were incubated in the plate for $30 \mathrm{~min}$ at $37^{\circ} \mathrm{C}$ and then washed with phosphate-buffered saline (PBS) 5 times for $15 \mathrm{sec}$ for each time, iv) enzymeconjugated reagent $(50 \mu \mathrm{l})$ was added and incubated at $37^{\circ} \mathrm{C}$ for $30 \mathrm{~min}$, followed by four $15-\mathrm{sec}$ washes and v) color developer A and B were added and the samples were incubated at room temperature in the dark for $15 \mathrm{~min}$. The termination solution was then added, and the OD value at $450 \mathrm{~nm}$ was measured using a microplate reader within $15 \mathrm{~min}$. These readings were used to calculate NGAL and Scr levels.

\section{Statistical analysis}

Date entry and data analysis were done using SPSS version 24 (Statistical Package for Social Science). Data were presented as number, percentage, mean and standard deviation. Chi-square test and Fisher Exact test were used to compare between qualitative variables. Independent sample t-test was used to compare quantitative variables between groups. P-value considered statistically significant when $\mathrm{P} \leq 0.05$.

\section{RESULTS}

Regarding demographic and clinical data, there were no significant differences $(\mathrm{P}>0.05)$ between the studied groups in age, gender, weight, height, BMI, type of operation, aortic cross clamp time or intraoperative UOP. While, there was a significant increase $(\mathrm{P}<0.05)$ in duration of $\mathrm{CPB}$ in the MUF group compared to non-MUF group as shown in table (1). 
Table (1): Demographic and clinical data of the studied groups

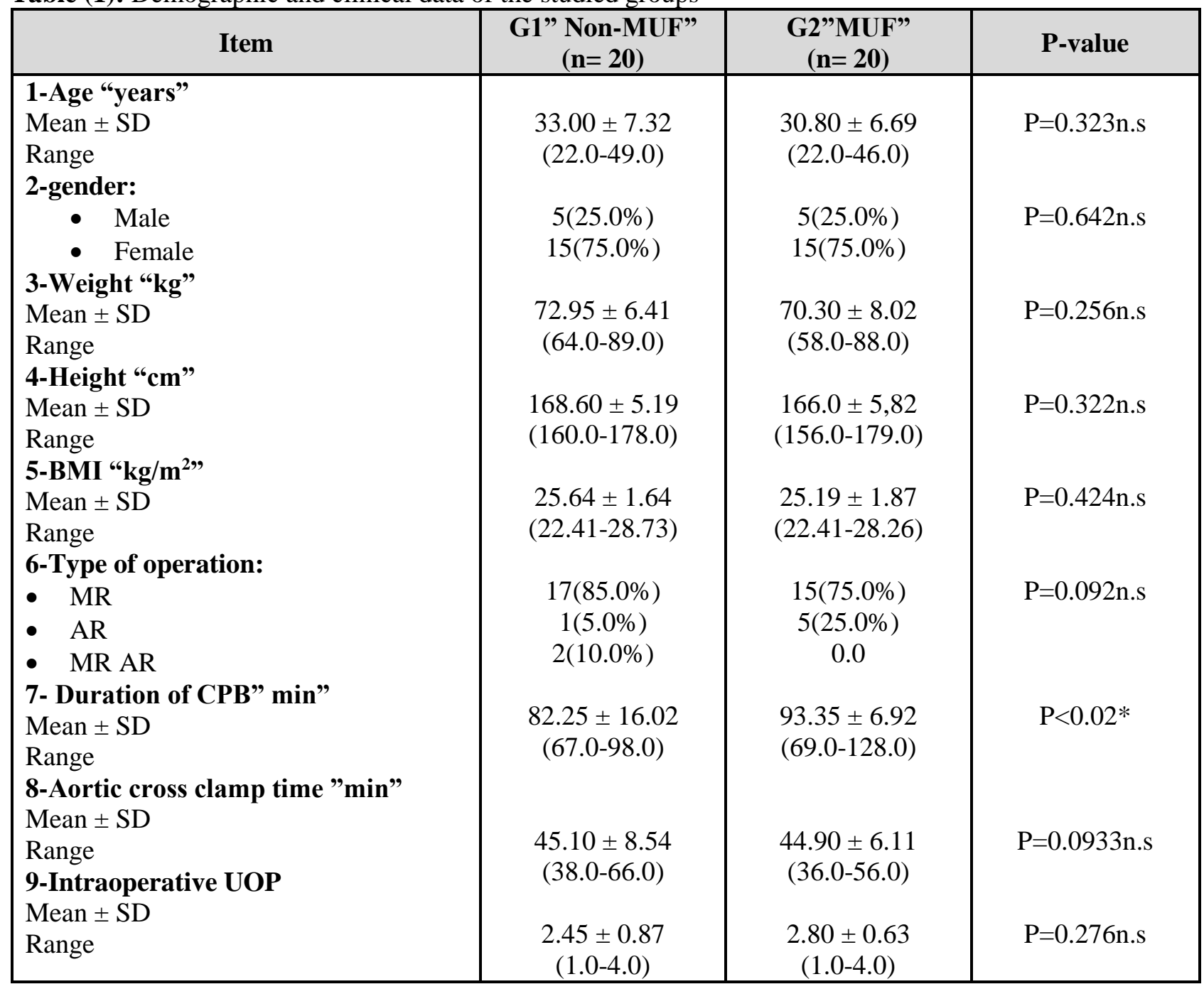

Abbreviations: Group1: without modified ultra-filtration; Group2: with modified ultrafiltration. BMI: body mass index, MR: Mitral valve replacement, AR: Aortic valve replacement, CPB: Cardiopulmonary bypass, UOP: Urine output. P value is considered significant at $\leq 0.05$

Effect of modified ultrafiltration on acute kidney injury biomarkers and clinical outcome According to KIDGO (Kidney Disease Improving Global Outcome) classification, patients in the MUF group were classified into AKI $\&$ non-AKI subgroups. Peri-operative AKI was graded using the KDIGO criteria as follows: grade 1: post-op serum creatinine $1.5-1.9$ times baseline or an increase in serum creatinine $\geq 26.5 \mu \mathrm{mol} / \mathrm{L}$, grade 2: postop serum creatinine 2.0-2.9 times baseline and grade 3: post-op serum creatinine 3 times baseline or increase in serum creatinine to $\geq 353.6 \mu \mathrm{mol} / \mathrm{L}$ or initiation of renal replacement therapy ${ }^{(9)}$.

\section{1- Regarding $S$. creatinine in the MUF group} There was no significant difference $(\mathrm{P}>0.05)$ between AKI and non-AKI patients in preoperative levels while the postoperative $S$. creatinine levels were significantly higher $(\mathrm{P}<$
0.04) in AKI than in non-AKI patients in the MUF group (Table 2).

Table (2): S. creatinine in the MUF group

\begin{tabular}{|l|c|c|c|}
\hline \multicolumn{1}{|c|}{ Item } & $\begin{array}{c}\text { AKI } \\
(\mathbf{n}=\mathbf{4})\end{array}$ & $\begin{array}{c}\text { Non AKI } \\
(\mathbf{n}=\mathbf{1 6})\end{array}$ & P-value \\
\hline $\begin{array}{l}\text { 1- Pre op. S. } \\
\text { creatinine }\end{array}$ & $0.95 \pm 0.05$ & $1.05 \pm 0.16$ & $\mathrm{P}=0.386 \mathrm{n} . \mathrm{s}$ \\
$\begin{array}{l}\text { 2- 2h Post op. } \\
\text { S. creatinine } \\
(\mu \mathrm{mol} / \mathrm{L})\end{array}$ & & $1.16 \pm 0.11$ & $\mathrm{P}<0.04 * 0.35$ \\
\hline
\end{tabular}

Abbreviations; AKI: Acute Kidney Injury; P value is considered significant at $<0.05$

2- Regarding S. NGAL in the MUF group.

The pre-operative and $2 \mathrm{~h}$ post-operative $\mathrm{S}$. NGAL was significantly higher in AKI than in non-AKI patients in the MUF group (Table 3). 
Table (3): NGAL the MUF group

\begin{tabular}{|l|c|c|c|}
\hline \multicolumn{1}{|c|}{ Item } & \multicolumn{1}{|c|}{$\begin{array}{c}\text { AKI } \\
(\mathbf{n}=\mathbf{4})\end{array}$} & $\begin{array}{c}\text { Non AKI } \\
(\mathbf{n = 1 6})\end{array}$ & P-value \\
\hline $\begin{array}{l}\text { 1-pre op. serum NGAL } \\
\text { (ng/ml) Mean } \pm \text { SD }\end{array}$ & $65.00 \pm 24.74$ & $39.75 \pm 2.14$ & $\mathrm{P}<0.001$ \\
$\begin{array}{l}\text { 2-2h.Post op. serum NGAL } \\
\text { (ng/ml) }\end{array}$ & $208.75 \pm 80.28$ & $77.25 \pm 21.94$ & $\mathrm{P}<0.0001$ \\
Mean \pm SD & & & \\
\hline
\end{tabular}

Abbreviations; AKI: Acute Kidney Injury; NGAL: Serum Neutrophil Gelatinase-Associated Lipocalin; P value is considered significant at $<0.05$

\section{3- Duration of CPB and Aortic cross clamp time of the MUF group.}

Duration of CPB was significantly prolonged $(\mathrm{P}<0.001)$ in the AKI than the in non-AKI patients. While, there was no significant difference regarding aortic cross-clamp time (Table 4).

Table (4): Duration of CPB and Aortic cross clamp time of the MUF group

\begin{tabular}{|l|c|c|c|}
\hline \multicolumn{1}{|c|}{ Item } & $\begin{array}{c}\text { AKI } \\
(\mathbf{n}=\mathbf{4})\end{array}$ & $\begin{array}{c}\text { Non AKI } \\
(\mathbf{n}=\mathbf{1 6})\end{array}$ & P-value \\
\hline 1-Duration of CPB" min" & $94.40 \pm 28.53$ & $78.20 \pm 6.75$ & $\mathrm{P}<0.001 * *$ \\
2-Aortic cross-clamp time "min" & $50.0 \pm 14.23$ & $43.46 \pm 5.46$ & $\mathrm{P}=0.438$ \\
\hline
\end{tabular}

Abbreviations: AKI: Acute Kidney Injury; CBP: Cardiopulmonary bypass output; P value is considered significant at $<0.05$

4-Iintraoperative UOP \& blood component requirements in the MUF group There was a significant higher fresh blood, packed RBCs unit requirements in the AKI patients and a significant decreased intraoperative UOP in the same patients (Table 5).

Table (5): Intraoperative blood component requirements and intraoperative UOP in the MUF group

\begin{tabular}{|l|c|c|c|}
\hline \multicolumn{1}{|c|}{ Item } & \multicolumn{1}{|c|}{$\begin{array}{c}\text { AKI } \\
(\mathbf{n = 4})\end{array}$} & $\begin{array}{c}\text { Non AKI } \\
(\mathbf{n = 1 6 )}\end{array}$ & P-value \\
\hline $\begin{array}{l}\text { 1-Fresh blood units } \\
\text { intraoperative }\end{array}$ & $1.25 \pm 0.47$ & $0.62 \pm 0.20$ & $\mathrm{P}<0.02^{*}$ \\
$\begin{array}{l}\text { 2-Packed RBCs units } \\
\begin{array}{l}\text { 3-Intraoperative } \\
\text { (mI/kg/hour) }\end{array}\end{array}$ & $2.00 \pm 0.81$ & $0.62 \pm 0.23$ & $\mathrm{P}<0.02^{*}$ \\
\hline
\end{tabular}

Abbreviations: AKI: Acute Kidney Injury; RBCs: Red Blood Cells; UOP: Urine output; P value is considered significant at $<0.05$

\section{5-Postoperative ICU stay \& Mechanical Ventilation in the MUF group}

There was a significant longer ICU stay and duration of mechanical ventilation in AKI than in non-AKI patients (Table 6).

Table (6): postoperative ICU stay \& Mechanical Ventilation in the MUF group

\begin{tabular}{|l|c|c|c|}
\hline \multicolumn{1}{|c|}{ Item } & $\begin{array}{c}\text { AKI } \\
(\mathbf{n = 4})\end{array}$ & $\begin{array}{c}\text { Non AKI } \\
(\mathbf{n = 1 6})\end{array}$ & P-value \\
\hline 1-ICU stay "days" & $3.00 \pm 1.41$ & $1.43 \pm 0.20$ & $\mathrm{P}<0.04^{*}$ \\
2-Duration of Mechanical & $4.50 \pm 1.25$ & $2.56 \pm 0.28$ & $\mathrm{P}<0.03^{*}$ \\
Ventilation "hours" & & & \\
\hline
\end{tabular}

Abbreviations: AKI: Acute Kidney Injury; ICU: Intensive Care Unit; P value is considered significant at $<0.05$

Correlation between 2h postoperative S. NGAL as an AKI Biomarkers with other variables in MUF group:

$2 \mathrm{~h}$ post-operative s. NGAL had positive correlation with each of $2 \mathrm{~h}$ post-operative $\mathrm{S}$. creatinine, packed RBCs, ICU stay and duration of mechanical ventilation, while had negative correlation with intraoperative UOP as shown in table (7). 
Table (7): Correlation between NGAL \& other variable in MUF group

\begin{tabular}{|c|c|c|}
\hline & & NGAL post-operative \\
\hline \multirow{2}{*}{ NGAL pre-operative } & $\mathrm{r}$ & \\
\hline & $p$ & \\
\hline \multirow{2}{*}{ NGAL post-operative } & $\mathrm{r}$ & 1 \\
\hline & $\mathrm{p}$ & \\
\hline \multirow{2}{*}{ Serum creatinine pre-operative } & $\mathrm{r}$ & -.085 \\
\hline & $p$ & .722 \\
\hline \multirow{2}{*}{ Serum creatinine post-operative } & $\mathrm{r}$ & .890 \\
\hline & $\mathrm{p}$ & .000 \\
\hline \multirow{2}{*}{ Duration of CPB min } & $\mathrm{r}$ & .200 \\
\hline & $p$ & .398 \\
\hline \multirow{2}{*}{ Aortic cross clamp time min } & $\mathrm{r}$ & .342 \\
\hline & $\mathrm{p}$ & .140 \\
\hline \multirow{2}{*}{ Fresh blood units intraoperatively } & $\mathrm{r}$ & -.002 \\
\hline & $\mathrm{p}$ & .994 \\
\hline \multirow{2}{*}{ Packed RBCs units } & $\mathrm{r}$ & .609 \\
\hline & $\mathrm{p}$ & .004 \\
\hline \multirow{2}{*}{ Intraoperative UOP } & $\mathrm{r}$ & -.768 \\
\hline & $\mathrm{p}$ & .000 \\
\hline \multirow{2}{*}{ ICU stay (days) } & $\mathrm{r}$ & .884 \\
\hline & $\mathrm{p}$ & .000 \\
\hline \multirow{2}{*}{ Mechanical ventilation (hours) } & $\mathrm{r}$ & .807 \\
\hline & $p$ & .000 \\
\hline
\end{tabular}

\section{DISCUSSION}

Cardiac surgery has long been used to study AKI because of the opportunity to prospectively follow patients before and after a well-timed ischemic renal insult (cardiopulmonary bypass). For this reason, several blood and urinary proteins have been investigated in this setting and found to serve as biomarkers of AKI after cardiac surgery, including neutrophil gelatinase-associated lipocalin (NGAL), IL-18, cystatin C (Cys C), kidney injury molecule-1 (KIM-1) and liver fatty acid binding protein ${ }^{(\mathbf{1 0})}$.

Our study found the pre-operative, $2 \mathrm{~h}$ postoperative S. NGAL and the postoperative $\mathrm{S}$. creatinine were significantly higher in AKI than in non-AKI patients in the MUF group. This agrees with Vives and his colleagues ${ }^{(11)}$ who reported that Patients with an early higher urinary and plasma NGAL levels are most likely to be diagnosed as AKI. NGAL has been demonstrated to be a highly sensitive and specific predictor of CSA-AKI. Urinary NGAL was demonstrated as an early biomarker of AKI after CPB, increasing 25fold within $2 \mathrm{~h}$ and declining $6 \mathrm{~h}$ after surgery. This promoted the use of urine NGAL as an indicator to forecast subclinical CSA-AKI ${ }^{(12)}$. McIlroy and his colleagues ${ }^{(12)}$ in a multicenter-pooled analysis of prospective studies showed that in the absence of diagnostic increases in S. creatinine, NGAL detected patients with likely subclinical AKI who have an increased risk of adverse outcomes. Therefore, NGAL levels may detect AKI 24-72 hrs earlier than S. creatinine and has a prognostic impact. Additionally, data from a meta-analysis on 2,322 patients suffering from AKI related to cardiac disease, suggested that patients with higher NGAL are most likely to develop adverse outcomes, even when S. creatinine is normal. Hence, it is suggested that subclinical AKI may be diagnosed by this biomarker ${ }^{(13)}$. Fadel et al. ${ }^{(14)}$ reported that mean plasma NGAL levels showed highly significant elevations at 2, 12, and $24 \mathrm{~h}$ after surgery $(\mathrm{p}<0.0001)$ in patients who developed AKI compared to patients who did not and significant correlations $(\mathrm{p}<0.0001)$ were found between plasma NGAL and AKI at 2, 12 and $24 \mathrm{~h}$ after surgery. A cut-off level of $100 \mathrm{ng} / \mathrm{ml}$ at $2 \mathrm{~h}$, and $125 \mathrm{ng} / \mathrm{ml}$ at $12 \mathrm{~h}$ post-operatively both recorded the highest accuracy, being $95 \%$ accurate, with sensitivity of $100 \%$ and $89.5 \%$ respectively, and specificity of $90.5 \%$ and $100 \%$ respectively. Vanmassenhove et al. ${ }^{(15)}$ in a recent meta-analysis that included 74 studies, identified NGAL as the biomarker with best discriminative performance for early diagnosis of cardiac surgery associated AKI and for outcome prediction.

In our results, there was a significant decreased intraoperative urine output in the AKI patients compared to non-AKI patients, which 
disagrees with Karim and his colleagues ${ }^{(16)}$ who reported in their study that the mean intraoperative urine output was not different in CSA-AKIpositive and -negative cases. This might be attributed to using a different perfusionist protocols.

In the our study, the $2 \mathrm{~h}$ post-operative s. NGAL had positive correlation with each of $2 \mathrm{~h}$ post-operative S. creatinine, packed RBCs, ICU stay and duration of mechanical ventilation, while had negative correlation with intraoperative UOP. This agrees with Koyner and Parikh ${ }^{(17)}$ who reported elevated urine and plasma NGAL levels that were all separately associated with longer length of hospital stay, longer ICU stay and higher risk for dialysis or death.

Regarding demographic and baseline hemodynamic data, the results showed no significant difference between the two study groups. Moreover, our results revealed a significant difference $(\mathrm{P}<0.05)$ between "MUF" and non-"MUF" group regarding used units of packed RBCs with lower requirement in "MUF" group than in non-"MUF" group. This agrees with Ziyaeifard et al. (18) who reported that MUF improved the hemostasis in the post-CPB period with useful effects on the blood loss, chest tube drainage, and blood transfusion requirements after the surgery. These results also are comparable to Kamada et al. ${ }^{(19)}$ who reported in their study that the need for blood products including red blood cells, fresh frozen plasma, cryoprecipitates and platelets were considerably lesser in MUF patients compared to the control group of patients.

Our study found no significant difference between the MUF and non-MUF groups in each of ICU stay \& duration of mechanical ventilation. However, there was a significant longer ICU stay and duration of mechanical ventilation in AKI patients compared to non-AKI patients in the MUF group. This agrees with Parolari et al. ${ }^{(20)}$ who reported that AKI patients had longer hospital and ICU stays.

Additionally, results of different studies demonstrated that the use of MUF in the post-CPB period in pediatrics patients can improve the pulmonary compliance and gas exchange, which may successfully reduce the pulmonary dysfunction after the pediatric cardiac surgery. MUF may lead to decreased period of tracheal intubation and mechanical ventilation, ICU stay and total hospitalization after the surgery ${ }^{(\mathbf{1 8})}$.

Our study found that duration of CPB is significantly prolonged in the AKI than in the nonAKI patients, while there was no significant difference regarding aortic cross-clamp time, which does not cope with Karim et al. ${ }^{(16)}$ who found that CSA-AKI increases with increasing both CPB and cross clamp time. That could be explained that in our research, we studied specific type of cardiac surgery with average ischemic time in contrast to wide varieties with largely variable ischemic times.

\section{CONCLUSION}

1. AKI to be a common and important complication of cardiac surgery and is associated with increased mortality, complications and length of hospital stay.

2. Modified ultrafiltration after cardiac surgery is associated with a lower prevalence of morbidity and mortality.

3. S. NGAL can be used as an early plasma biomarker predictor for AKI in patients undergoing heart surgery.

\section{REFERENCES}

1. Uchino S, Kellum JA, Bellomo $\mathrm{R}$ et al. (2005): Beginning, Ending Supportive Therapy for the Kidney I: Acute renal failure in critically ill patients: a multinational, multicenter study. JAMA., 294: 813-818.

2. Bellomo R, Auriemma S, Fabbri A et al. (2008): The pathophysiology of cardiac surgery-associated acute kidney injury (CSA-AKI). Int J Artif Organs, 31: 166-178.

3. Zakeri R, Freemantle N, Barnett V et al. (2005): Relation between mild renal dysfunction and outcomes after coronary artery bypass grafting. Circulation, 112: 270-275.

4. Foroughi M, Argani H, Hassntash SA et al. (2014): Lack of renal protection of ultrafiltration during cardiac surgery: a randomized clinical trial. J Cardiovasc Surg., 55: 407-13.

5. Kuntz RA, Holt DW, Turner S et al. (2006): Effects of conventional ultrafiltration on renal performance during adult cardiopulmonary bypass procedures. J Extra Corpor Technol., 38: 144-53.

6. Boyle A, Maurer MS, Sobotka PA (2007): Myocellular and interstitial edema and circulating volume expansion as a cause of morbidity and mortality in heart failure. J Card Fail., 13: 133-6.

7. Luciani GB, Menon T, Vecchi B et al. (2001): Modified ultrafiltration reduces morbidity after adult cardiac operations: a prospective, randomized clinical trial. Circulation, 104 (12-1): 253-259.

8. Keenan HT, Thiagarajan R, Stephens KE et al. (2000): Pulmonary function after modified venovenous ultrafiltration in infants: a prospective, randomized trial. J Thorac Cardiovasc Surg., 119: 501-505.

9. Kellum JA, Lameire N (KDIGO AKI Guideline Work Group) (2013): Diagnosis, evaluation, and management of acute kidney injury: a KDIGO summary (Part 1). Crit Care, 17: 204.

10. Liangos $\mathrm{O}$, Tighiouart $\mathrm{H}$, Perianayagam $\mathrm{M}$ et al. (2009): Comparative analysis of urinary 
biomarkers for early detection of acute kidney injury following cardiopulmonary bypass. Biomarkers, 14: 423-431.

11. Vives M, Hernandez A, Parramon F et al. (2019): Acute kidney injury after cardiac surgery: prevalence, impact and management challenges. Int J Nephrol Renovasc Dis., 12: 153-166.

12. McIlroy D, Wagener G, Lee H (2010): Neutrophil gelatinase-associated lipocalin and acute kidney injury after cardiac surgery: the effect of baseline renal function on diagnostic performance. Clin $\mathbf{J}$ Am Soc Nephrol., 5: 211-219.

13. Haase M, Devarajan $P$, Haase-Fielitz A et al. (2011): The outcome of neutrophil gelatinaseassociated lipocalin-positive subclinical acute kidney injury: a multicenter pooled analysis of prospective studies. J Am Coll Cardiol., 57 (17): 1752-61.

14. Fadel F, Abdel Rahman A, Mohamed M et al. (2012): Plasma neutrophil gelatinase-associated lipocalin as an early biomarker for prediction of acute kidney injury after cardio-pulmonary bypass in pediatric cardiac surgery. Arch Med Sci., 8 (2): 250-255.

15. Vanmassenhove J, Vanholder R, Nagler E et al. (2013): Urinary and serum biomarkers for the diagnosis of acute kidney injury: an in-depth review of the literature. Nephrol Dial Transplant, 28: 254273.

16. Karim H, Yunus M, Saikia M et al. (2017): Incidence and Progression of Cardiac Surgeryassociated Acute Kidney Injury and its Relationship with Bypass and Cross Clamp Time. Ann Card Anaesth., 20 (1): 22-27.

17. Koyner J, Parikh C (2013): Clinical utility of biomarkers of AKI incardiac surgery and critical illness. Clin J Am Soc Nephrol., 8: 1034-1042.

18. Ziyaeifard M, Alizadehas A, Massoumi G (2014): Modified Ultrafiltration During Cardiopulmonary Bypass and Postoperative Course of Pediatric Cardiac Surgery. Res Cardiovasc Med., 3 (2): 17830.

19. Kamada M, Niibori $K$, Akimoto $H$ et al. (2001): Efficacy of modified ultrafiltration in reoperation for valvular disease. Kyobu Geka., 54 (2): 119-24.

20. Parolari A, Pesce LL, Pacini D et al. (2012): Monzino Research Group on Cardiac Surgery Outcomes: Risk factors for perioperative acute kidney injury after adult cardiac surgery: Ann Thorac Surg., 93: 584-591. 